\title{
A Case of Erdheim-Chester Disease with Asymptomatic Renal Involvement
}

\author{
Hyun Jung Lee, MD ${ }^{1}$ \\ Kyoung Yul Lee, MD² \\ Dong-Yeop Shin, MD ${ }^{1}$ \\ Yun Gyoo Lee, MD \\ Se Youn Choi, MD' \\ Kyung Chul Moon, MD2 \\ II-Kyu Han, $\mathrm{MD}^{3}$ \\ Tae Min Kim, MD
}

Departments of ${ }^{1}$ Internal Medicine, ${ }^{2}$ Pathology, and ${ }^{3}$ Orthopedic Surgery, Seoul National University Hospital, Seoul National University College of Medicine, Seoul, Korea
Erdheim-Chester disease is a rare non-Langerhans-cell histiocytosis involving bones and multiple organs. Its clinical course can vary, from an asymptomatic state to a fatal disease, with renal involvement being a common cause of death. A41-year-old man presented with a 10-month history of bilateral lower limb pain. Left perirenal soft-tissue infiltration had been found incidentally two years earlier. No progression of the lesion or deterioration of renal function was observed for a period of two years. At admission, plain radiography and magnetic resonance imaging of the patient's lower limbs showed patchy osteosclerosis. Biopsy of the tibia revealed histiocytic infiltration, which was found to be positive for $\mathrm{CD} 68$ and negative for $\mathrm{CD} 1 \mathrm{a}$. This report describes an unusual case of Erdheim-Chester disease involving a stationary course of disease with no specific treatment for a long period of time.

\section{Key words}

Non-Langerhans-cell histiocytosis, Osteosclerosis, Retroperitoneal fibrosis

\section{Introduction}

Erdheim-Chester disease (ECD) is a rare non-Langerhans-cell histiocytosis of unknown etiology. Since it was first described by Erdheim and Chester in 1930 [1], approximately 400 or more cases of ECD have been reported in the literature. Since the first case of ECD was described in 1999, 10 cases have been reported in Korea [2]. Age at onset is 40-60 years. The disease is characterized by infiltration of lipid-laden histiocyte-producing xanthogranulomas in long tubular bones, leading to osteosclerosis. Xan- thogranulomatous tissues can also be found in skin, lung, heart, kidney, retroperitoneal space, orbit, and pituitary gland. Bone pain is the most common symptom, and bilateral osteosclerosis of long bones, excluding the epiphysis, is regarded as a typical radiological finding [3]. Results of histopathological analysis show large, foamy or lipid-laden histiocytes with eosinophilic cytoplasm and/or pathognomonic Touton-like giant cells [4]. ECD is usually a progressive disease, with death from cardiac, pulmonary, or renal involvement occurring in approximately $50 \%$ of cases. We report on a patient with an indolent course of the disease, who presented with asymptomatic renal involvement followed by mild limb pain. 

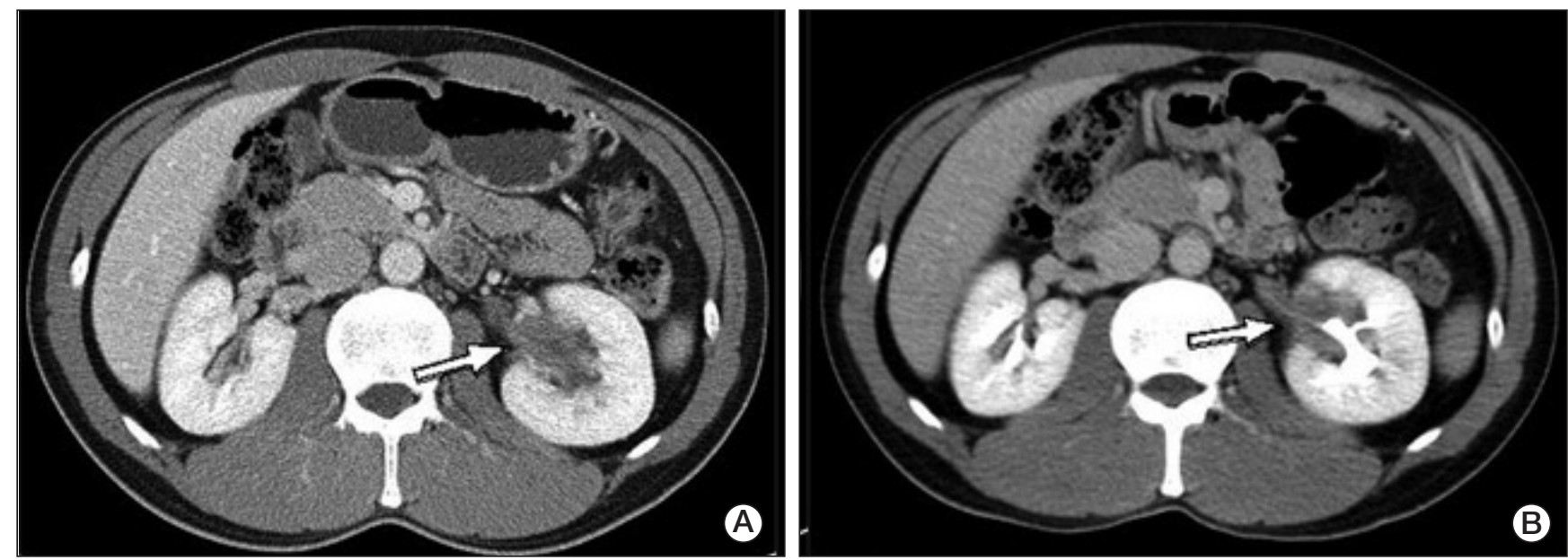

Fig. 1. Computed tomography (CT) scan of the patient's abdomen in 2008 showed tissue infiltration with low attenuation surrounding the left renal sinus and the proximal ureter (A). No significant change was observed on CT scan in 2010 (B).
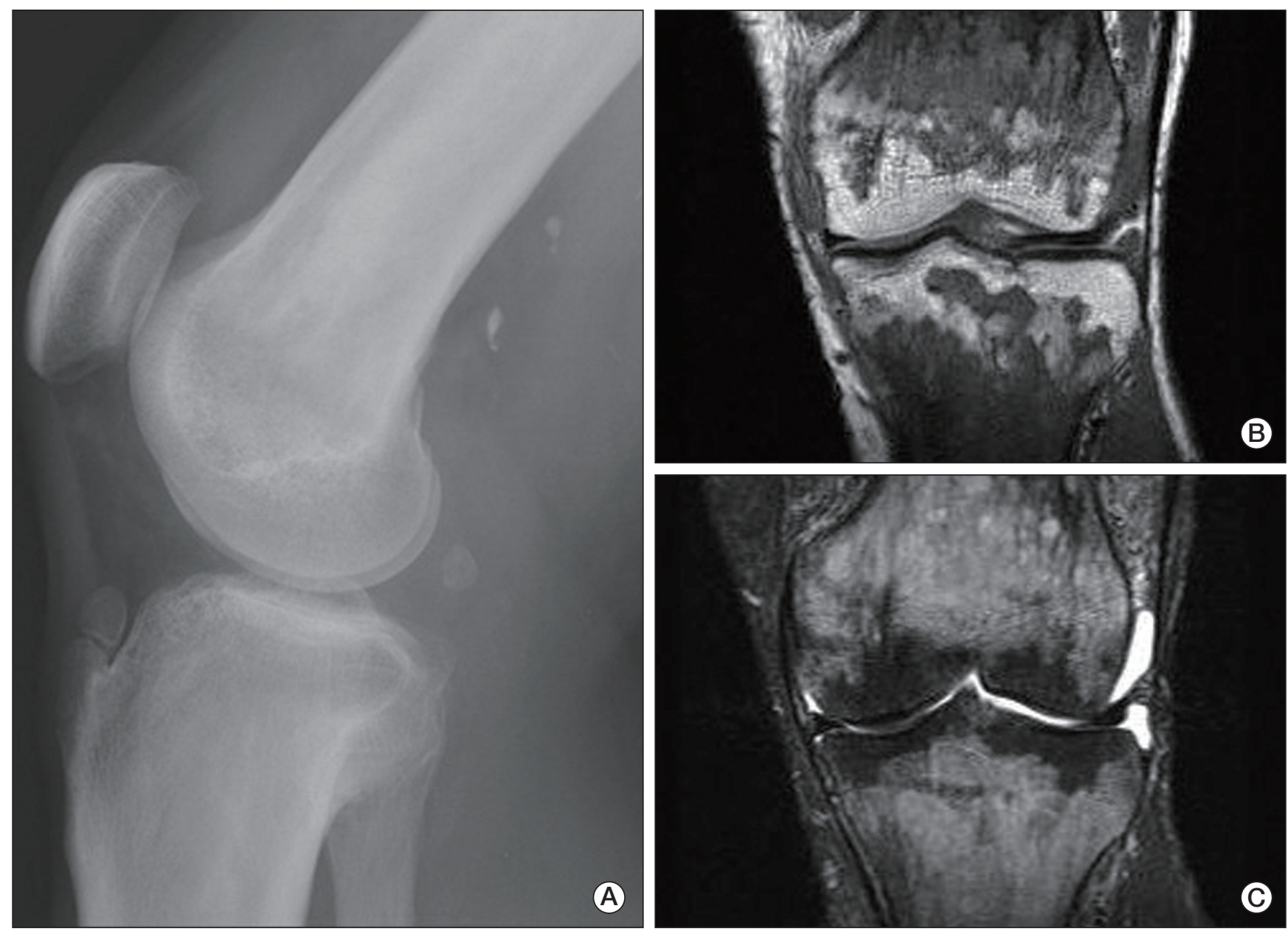

Fig. 2. Simple radiography of the left knee showed heterogeneous patchy medullary sclerosis, coarse trabeculae, and osteopenia of the covered femur and tibia (A). Magnetic resonance imaging of the knee showed diffuse sclerotic changes combined with intramedullary low signal intensity on a T1-weighted image (B) and mixed low- and high-signal nodular lesions on a T2-weighted image (C). Involvement of mainly the meta- and diaphysis, with relative epiphyseal sparing, was observed. 


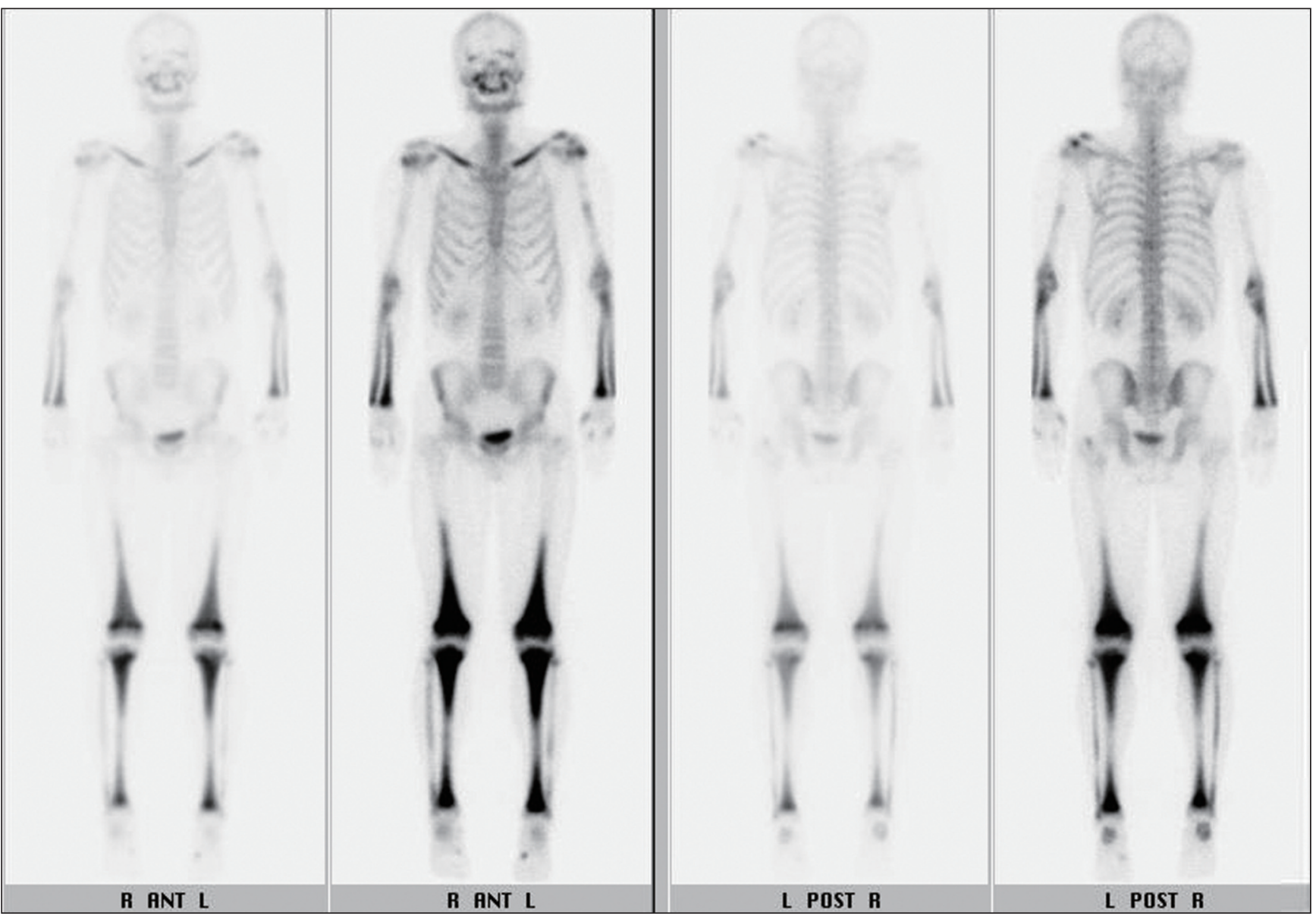

Fig. 3. Whole-body bone scintigraphy revealed diffuse increased uptake in the bilateral clavicles, radii, ulnae, distal femora, tibiae, and fibulae.
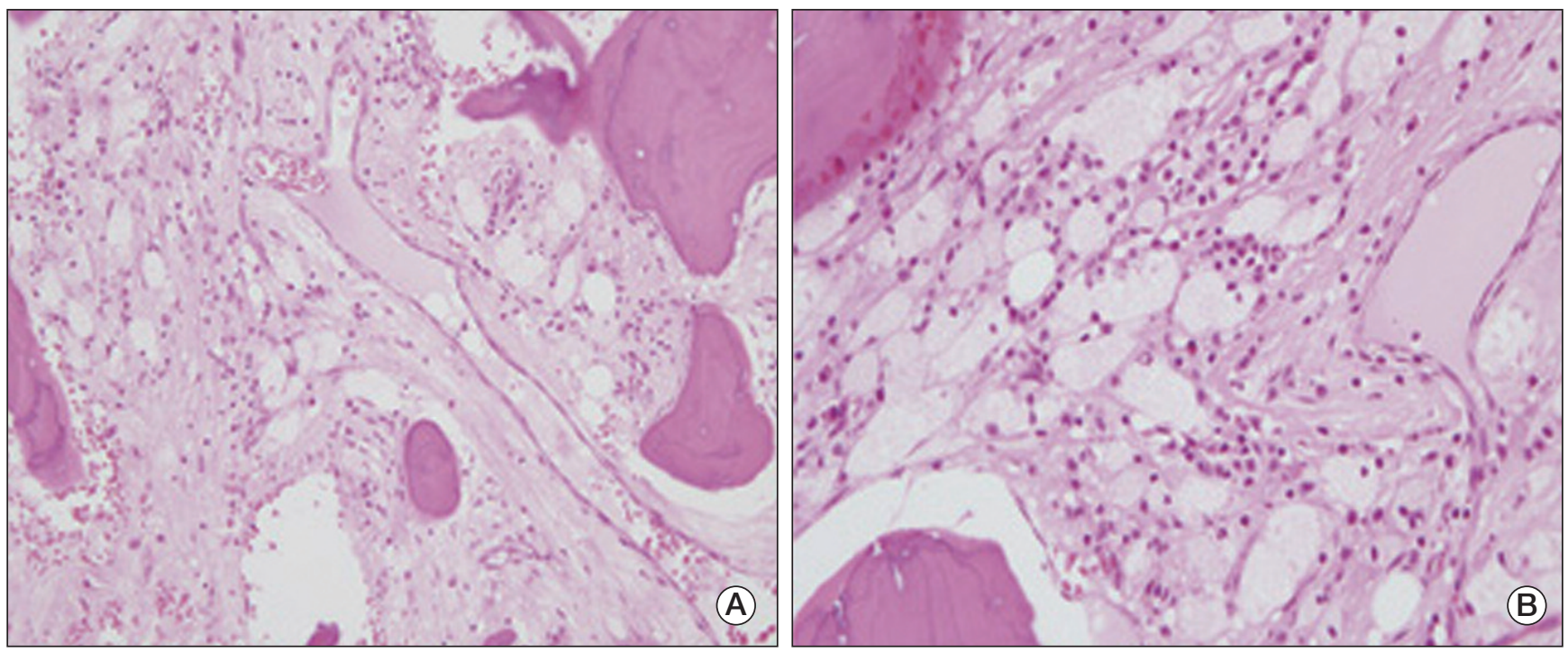

Fig. 4. Results of hematoxylin and eosin staining showed sclerotic bony trabeculae containing lipid-laden histiocytes and small histiocytes with oval nuclei and pink cytoplasm. A small number of infiltrating inflammatory cells were observed; however, eosinophils were rare $(\mathrm{A}, \times 200$; $\mathrm{B}, \times 400)$. 


\section{Case Report}

A 41-year-old man presented with left hydronephrosis, detected incidentally during a regular checkup in July 2008. A computed tomography (CT) scan of his abdomen showed an infiltrative homogeneous mass involving the left renal sinus, with low attenuation on CT (Fig. 1A). Results of a ureteroscopic biopsy revealed nonspecific inflammation. However, result of urine analysis and microscopic examination showed no renal dysfunction or abnormality. Until July 2010, follow-up CT scans showed no significant change in perirenal infiltration (Fig. 1B).

In March 2010, the patient was admitted to the hospital complaining of bone pain that had lasted for 10 months, with no significant history of trauma. He reported mild pain in both knees, which gradually extended to both ankles and both upper limbs. However, the pain he described had not affected his daily life. He showed no sign of fever or weight loss. His erythrocyte sedimentation rate was $23 \mathrm{~mm} / \mathrm{hr}$ and his C-reactive protein was $3.09 \mathrm{mg} / \mathrm{dL}$. Results of urine analysis, complete blood count, renal and liver function tests, and lipid profiles were normal. Plain radiography of the left knee (Fig. 2A) showed heterogeneous patchy medullary sclerosis and coarse trabeculae and osteopenia of the covered femur and tibia. Symmetric and diffuse sclerotic changes were observed on gadoliniumenhanced magnetic resonance imaging (MRI) of his knees and ankles. Involvement of mainly the meta- and diaphyses, with relative epiphyseal sparing, was observed (Fig. 2B and C). Diffuse and symmetric uptake in the bilateral tibiae, fibulae, distal femora, radii, ulnae, humeri, and clavicles was observed by whole-body bone scintigraphy (Fig. 3). MRI of the brain and CT scan of the chest showed no abnormalities. Biopsy of the proximal tibia revealed sclerotic bony trabeculae, with lipid-laden histiocytic and lymphocytic infiltration (Fig. 4). As a result of immunohistochemical staining, lipid-laden histiocytes were found to be positive for CD68 (Fig. $5 \mathrm{~A}$ ) and negative for S-100 and CD1a (Fig. 5B). Results of a bone marrow examination also revealed two focal lymphohistiocytic infiltrations, which were found to be positive for CD68 and negative for CD1a. Bone marrow examination showed no atypical cells. Bone pain described by the patient did not require intervention; therefore, a wait-and-see policy was adopted in the outpatient clinic.

\section{Discussion}

Occurrence of ECD is extremely rare and diagnosis is difficult; therefore, clinical suspicion is an important factor in its diagnosis. Final diagnosis is based on clinical suspicion, classic radiographic findings in long bones, and biopsy, with histological confirmation.

In diagnosis, ECD must be distinguished from other granulomatous disorders. Chronic recurrent multifocal osteomyelitis (CRMO) is of particular concern. CRMO was initially described as a symmetric osteomyelitis; however, since no causative organism has been identified, it is now considered a noninfectious inflammatory disease. The clinical course of CRMO is self limited. CRMO involves the mandible and spine and lesions are associated with local inflammation and prominent periosteal reaction. When involvement of multiple bones is detected, evaluation for tumors such as mastocytosis, myelofibrosis, lymphoma, and osteosclerotic myeloma should be performed. In these tumorous conditions, the presence of many systemic manifestations is observed more often, according to the situation [5].

Morphologically and immunohistochemically, ECD appears to be a member of the juvenile xanthogranuloma (JXG) group. JXG cells express factor XIIIa, CD68, and are negative for CD1a and Lagenrin. S-100 positivity is reported in less than $20 \%$ of cases. However, none of these markers is specific for JXG. Involvement of bilateral symmetric long bones in
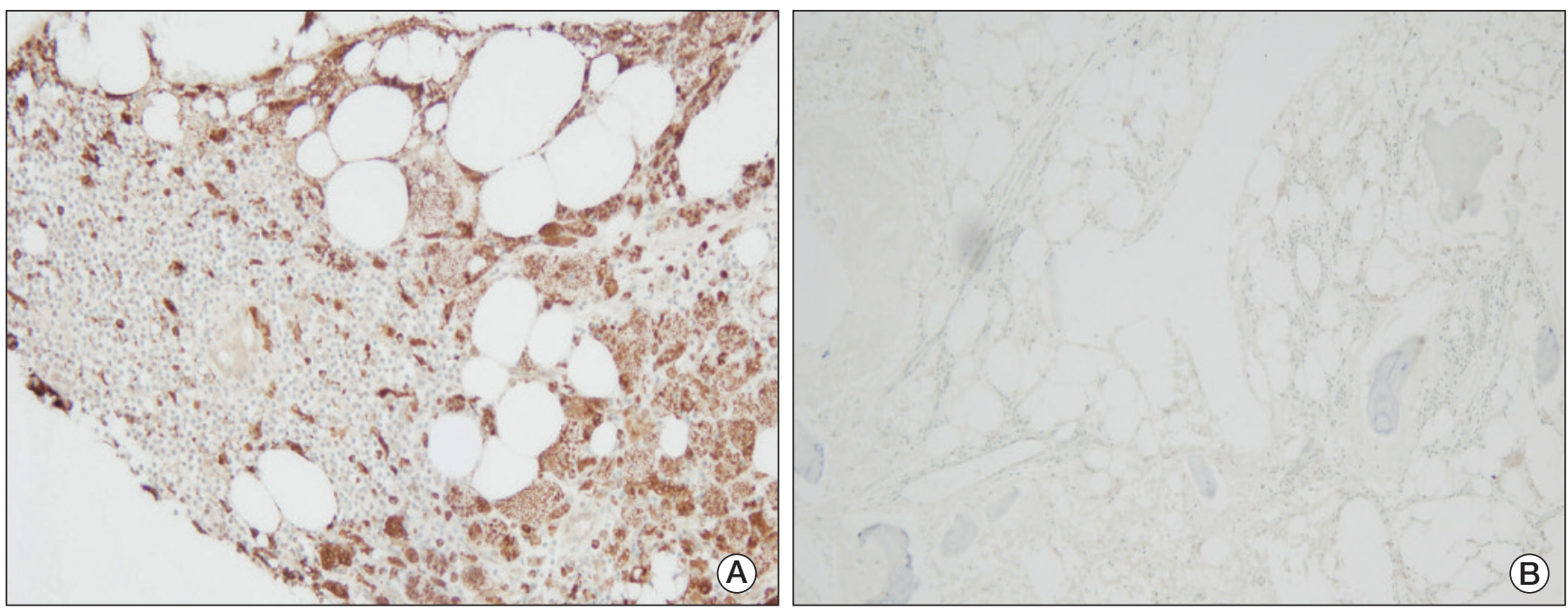

Fig. 5. Lipid-laden histiocytes were positive for CD68 (A, CD68 immunostain, $\times 100)$. Histiocytes were negative for CD1a (B, CD1a immunostain, $\times 200)$. 
association with extraskeletal organ involvement is a characteristic feature of ECD. ECD occurs mainly in middle aged adults, while other systemic JXG occurs mainly in young children [6].

In this patient, an incidentally detected perirenal infiltrative mass was the first manifestation of ECD. Bone lesions were detected after a twoyear asymptomatic period. Perirenal infiltration is a well-known extraskeletal finding of ECD. Veyssier-Belot et al. [3] reported renal and retroperitoneal involvement in 30\% of patients. Hydronephosis, an enlarged kidney, and retroperitoneal infiltration surrounding the abdominal aorta and kidneys were observed on abdominal $\mathrm{CT}$ in cases of renal and retroperitoneal involvement of ECD. This involvement was usually asymptomatic and CT scan revealed hypoattenuated homogenous tissue infiltration [3]. Although CT images of the current case showed only mild hydronephrosis, the nature of the tissue surrounding the left kidney and ureter was consistent with that of retroperitoneal involvement of ECD; the present case demonstrated typical bone involvement of ECD. Therefore, despite the lack of pathologic confirmation, we suggested that the perirenal infiltrative mass could be considered renal involvement of ECD.

The clinical course of ECD varies. According to the extent of organ involvement, patients present with a range of symptoms extending from a focal asymptomatic state to multisystemic, fatal, infiltrative disease. Primary causes of death include cardiomyopathy, interstitial lung disease, and renal failure. Unfortunately, approximately $60 \%$ of patients died of ECD within three years from initial presentation [3]. No treatment for ECD has yet been established; however, treatment of ECD patients can include steroids, cytotoxic agents [3], bisphosphonate [7], and autologous stem cell transplantation [8]. Interferon- $\alpha$ (IFN- $\alpha)$ as a valuable therapy for treatment of patients with ECD, with tolerable toxicity [9-11], and long-lasting responses (3-4 years), has recently been reported.

Renal failure can be attributed to obstructive uropathy in association with retroperitoneal fibrosis (79\%) or renal histiocytic infiltration (21\%) [12]. However, renal lesions are not responsive to systemic immunosuppressive agents, whereas bone pain and general performance status show improvement with treatment. Most patients with renal involvement die within five years of diagnosis [13]. IFN- $\alpha$ is the only effective agent known to stabilize retroperitoneal fibrosis and cause its regression.

Efficacy of IFN- $\alpha$ varies according to the site of involvement. Treatment with IFN- $\alpha$ appears to be effective in patients with renal involvement, whereas its failure has been reported in patients with severe multisystemic involvement that includes the central nervous system and cardiovascular system [9]. Early therapeutic intervention with INF- $\alpha$ appears to be effective in preventing progression of cardiovascular and renal involvement [11]. Imatinib mesylate has been suggested by some investigators as an experimental alternative therapeutic regimen after failure of INF- $\alpha[14]$.

Most patients with renal involvement show a poor response to treatment. Approximately half develop renal insufficiency and die within two years, on average [12]. The patient described here developed a new bone lesion within a period of two years; however, his renal lesion did not show progression. Although a follow-up period of two years is short, a waitand-see policy can be adopted in ECD patients with an asymptomatic renal lesion.

\section{Conflicts of Interest}

Conflicts of interest relevant to this article was not reported.

\section{References}

1. Chester W. Über lipoidgranulomatose. Virchows Arch Pathol Anat Physiol. 1930;279:561602.

2. Park YK, Ryu KN, Huh B, Kim JD. Erdheim-Chester disease: a case report. J Korean Med Sci. 1999;14:323-6.

3. Veyssier-Belot C, Cacoub P, Caparros-Lefebvre D, Wechsler J, Brun B, Remy M, et al. Erdheim-Chester disease. Clinical and radiologic characteristics of 59 cases. Medicine (Baltimore). 1996;75:157-69.

4. Zelger B, Cerio R, Orchard G, Wilson-Jones E. Juvenile and adult xanthogranuloma. A histological and immunohistochemical comparison. Am J Surg Pathol. 1994;18:126-35.

5. Case records of the Massachusetts General Hospital. Weekly clinicopathological exercises. Case 9-2000. A 41-year-old man with multiple bony lesions and adjacent soft-tissue masses. N Engl J Med. 2000;342:875-83.

6. Weitzman S, Jaffe R. Uncommon histiocytic disorders: the non-Langerhans cell histiocytoses. Pediatr Blood Cancer. 2005:45:256-64

7. Mossetti G, Rendina D, Numis FG, Somma P, Postiglione L, Nunziata V. Biochemical markers of bone turnover, serum levels of interleukin-6/interleukin-6 soluble receptor and bisphosphonate treatment in Erdheim-Chester disease. Clin Exp Rheumatol. 2003;21:232-6.

8. Boissel N, Wechsler B, Leblond V. Treatment of refractory Erdheim-Chester disease with dou- ble autologous hematopoietic stem-cell transplantation. Ann Intern Med. 2001;135:844-5

9. Haroche J, Amoura Z, Trad SG, Wechsler B, Cluzel P, Grenier PA, et al. Variability in the efficacy of interferon-alpha in Erdheim-Chester disease by patient and site of involvement: results in eight patients. Arthritis Rheum. 2006;54:3330-6.

10. Braiteh F, Boxrud C, Esmaeli B, Kurzrock R. Successful treatment of Erdheim-Chester disease, a non-Langerhans-cell histiocytosis, with interferon-alpha. Blood. 2005;106:2992-4.

11. Suzuki HI, Hosoya N, Miyagawa K, Ota S, Nakashima H, Makita N, et al. Erdheim-Chester disease: multisystem involvement and management with interferon-alpha. Leuk Res. 2010:34:e21-4.

12. Sanchez JE, Mora C, Macia M, Navarro JF. Erdheim-Chester disease as cause of end-stage renal failure: a case report and review of the literature. Int Urol Nephrol. 2010;42:1107-12.

13. Mounach A, Nouijai A, Achemlal L, El Maghraoui A, Bezza A. Erdheim-Chester disease: a case report with pulmonary, kidney involvement and bone lesions. Rheumatol Int 2010:30:651-4.

14. Haroche J, Amoura Z, Charlotte F, Salvatierra J, Wechsler B, Graux C, et al. Imatinib mesylate for platelet-derived growth factor receptor-beta-positive Erdheim-Chester histiocytosis. Blood. 2008:111:5413-5. 